\title{
Estabilidad oxidativa de huevos enriquecidos con ácidos grasos poliinsaturados omega 3 , frente a antioxidantes naturales
}

\author{
María Elena Bernal Gómez¹, Cássio Xavier de Mendonça-Junior², Jorge Mancini-Filho*
}

'Departamento de Alimentos e Nutrição Experimental, Faculdade de Ciências Farmacêuticas, Universidade de São Paulo, ²Departamento de Clínica Médica, Faculdade de Medicina Veterinária e Zootecnia, Universidade de São Paulo

*Correspondencia:

J. Mancini-Filho

Departamento de Alimentos e

Nutrição

FCF, USP

Av. Lineu Prestes 580, Bloco 14, Cidade Universitária

05508-900, São Paulo-SP, Brasil Email: jmancini@usp.br
Fueron alimentadas 192 gallinas ponedoras de 22 semanas de edad, de linaje comercial Babcock, durante 30 dias con dietas de 0 (cero) y 12,7\% de semilla de linaza molida que corresponde a 0 y $5 \%$ de aceite de linaza, respectivamente. Fueron definidos 8 tratamientos: 4 grupos con 12,7\% de semilla de linaza (control/sin antioxidante; BHA + BHT, 100+100 ppm; orégano, 200 ppm; romero, 200 ppm) y 4 grupos sin semilla de linaza, pero utilizando los mismos antioxidantes. Este estudio fue realizado con el objetivo de verificar la eficacia del uso de antioxidantes naturales provenientes del orégano y del romero en la protección contra el deterioro oxidativo de la fracción lipídica de las yemas de los huevos enriquecidos con ácidos grasos poliinsaturados omega 3 (PUFA $\omega-3$ ). El grado de oxidación lipídica fue determinado a través de la prueba TBARS (substancias reactivas con ácido tiobarbitúrico). Según los resultados obtenidos, fue verificada diferencia significativa en la reducción del grado de oxidación lipídica de las yemas de huevo en todos los tratamientos con antioxidantes, cuando se compara con el control. Por lo tanto, los extratos de los condimentos, romero y orégano, pueden ser utilizados satisfactoriamente para obtener huevos enriquecidos con PUFA $\omega-3$, mejorando la estabilidad lipídica.
Unitermos

- Peroxidación lipídica

- Ácidos grasos poliinsaturados omega 3

- Antioxidantes naturales

\section{INTRODUCCIÓN}

La oxidación lipídica, al ser un fenómeno espontáneo e inevitable, implicando directamente en el valor comercial sea de los compuestos grasos, sea de todos los productos que a partir de ellos son formulados (Silva et al., 1999), conduce a cambios que ocurren durante el proceso, distribución y preparación final de los alimentos. La oxidación de lípidos inicia también otros cambios en los alimentos que afectan su cualidad nutricional, seguridad, color, flavor y textura (Shahidi, Wanasundara, 1992). Este aspecto es de gran importancia, no solamente bajo el enfoque económico, a través de pérdidas debido a la disminución de la vida útil, sino también por la posibilidad de que los radicales libres formados reaccionen con otros constituyentes de los alimentos provocando una reducción 
en la calidad nutricional de los mismos (Nawar, 1996).

Los ácidos grasos esterificados con el glicerol, formando los triacilglicéridos, son los principales componentes de la fracción lipídica. Entre los ácidos grasos están presentes estructuras insaturadas que son más susceptibles a la oxidación de que las saturadas. Los ácidos grasos insaturados pueden presentar una insaturación o más, donde se encuentran los ácidos grasos poliinsaturados (PUFA) que presentan menor estabilidad oxidativa que los ácidos grasos monoinsaturados (Nawar, 1996).

La incorporación de los ácidos grasos de la serie w3 en huevos puede ser hecha a través del proveimiento de la ración para aves (Marshall et al., 1994). Sin embargo, por el número de insaturación, los PUFA son más propensos al ataque de radicales libres (Papas, 1999) y al daño oxidativo, siendo utilizados en la determinación de la eficacia de antioxidantes naturais (Wanasundara, Shahidi, 1998), con el aumento de estos ácidos en las dietas de las aves, hay um aumento concomitante en la susceptibilidad al deterioro oxidativo de los huevos, llevando a la pérdida de las características tanto de la calidad como del valor nutritivo, menor aceptación del consumidor y efectos biológicos adversos (Bernal et al., 2002; López Bote et al., 1998).

En la ausencia de antioxidantes apropiados, los PUFA forman radicales libres y pueden tener un efecto prooxidante significativo llevando a la pérdida de la vitamina $\mathrm{E}$ y aumento de los productos de oxidación (Meydani, 1996). Por consiguiente, es un requisito necesario tener un consumo mayor de antioxidantes para acompañar un consumo elevado de ácidos grasos poliinsaturados y obtener así, la acción benéfica de los mismos (Wiseman, 1996).

Muchos antioxidantes sintéticos como butilhidroxianisol (BHA), butilhidroxitolueno (BHT), galato de propilo (PG) y terbutilhidroquinona (TBHQ), presentan eficacia en la reducción de la oxidación lipídica a una concentración de 200 ppm. Antioxidantes naturales extraídos de plantas pueden ser usados como alternativas de los antioxidantes sintéticos, debido a su efecto equivalente o mayor en la inhibición de la oxidación lipídica (Namiki, 1990). Madsen y Bertelsen (1995) y Cintra y ManciniFilho (2001) indican algunos condimentos, como romero y orégano presentando actividad antioxidante eficaz.

\section{MATERIAL Y MÉTODOS}

\section{Material}

La semilla de linaza (Linun usitatissimum L.) y los condimentos, orégano (Origanum vulgare L.) y romero
(Rosmarinus officinalis L.) fueron adquiridas en el mercado de la ciudad de São Paulo, Brasil.

Los antioxidantes sintéticos utilizados fueron el butilhidroxianisol (BHA) y el butilhidroxitolueno (BHT), de las marcas Sigma y BDH Chemicals Ltd., respectivamente.

\section{Métodos}

Determinación del perfil de ácidos grasos de la linaza

La esterificación de los ácidos grasos del aceite extraído de la linaza por el método de Soxhlet, utilizándose como solvente éter etílico, fue realizada según la técnica propuesta por Hartman y Lago, 1973. Para esta determinación fue utilizado cromatógrafo gaseoso marca Shimadzu, modelo GC17A, con las siguientes condiciones cromatográficas:

Columna: capilar de sílica fundida, Carbowax 20M (30 m x $0,25 \mathrm{~mm}$ d.i)

Detector: ionización de llama

Programación de la columna: temperatura inicial de $80^{\circ} \mathrm{C}$, aumentar hasta $150{ }^{\circ} \mathrm{C}\left(10^{\circ} \mathrm{C} / \mathrm{min}\right)$. Aumentar la temperatura hasta $230{ }^{\circ} \mathrm{C}\left(6^{\circ} \mathrm{C} / \mathrm{min}\right)$ y mantener por $30 \mathrm{~min}$.

Gas de arrastre: helio, con flujo de $1 \mathrm{~mL} / \mathrm{min}$.

Temperatura del inyector: $250^{\circ} \mathrm{C}$.

Temperatura del detector: $250^{\circ} \mathrm{C}$.

Los metil ésteres de los ácidos grasos fueron identificados por comparación con los tiempos de retención de estándares.

\section{Preparación de las raciones}

Fueron preparadas 2 raciones isocalóricas e isoproteicas de acuerdo a las tablas NRC (National Research Council, 1994), conteniendo 0 (cero) y $12,7 \%$ de semilla de linaza molida, que corresponde a 0 y $5 \%$ de aceite de linaza, respectivamente, suplementadas a la dieta base de maíz y soya (Tabla I).

A partir de cada una de las raciones, fueron preparadas tres formulaciones conteniendo una mezcla de antioxidantes sintéticos: BHA 100 ppm + BHT 100 ppm, orégano 200 ppm y romero $200 \mathrm{ppm}$, separadamente, resultando en seis tratamientos, cada grupo con su respectivo control (sin antioxidante), así:

- Control $0 \%$ aceite de linaza (0\% linaza molida)

- $0 \%$ linaza + BHA / BHT (1)

- $0 \%$ linaza + orégano $(2)$

- $0 \%$ linaza + romero (3)

- Control 5\% aceite de linaza (12,6\% linaza molida)

- $5 \%$ linaza + BHA / BHT (4)

- $5 \%$ linaza + orégano $(5)$

- $5 \%$ linaza + romero $(6)$ 
TABLA I - Formulaciones de las raciones (\%)

\begin{tabular}{lcc}
\hline MATERIA PRIMA & $\begin{array}{c}\text { FORMULACIÓN I } \\
\text { 0\% semilla de linaza }\end{array}$ & $\begin{array}{c}\text { FORMULACIÓN II } \\
\mathbf{1 2 , 7 \%} \text { semilla de linaza }\end{array}$ \\
\hline Maíz & 55,84 & 49,74 \\
Salvado de soya-45 & 27,02 & 23,32 \\
Calcario & 9,94 & 9,88 \\
Aceite de maíz & 5,00 & 2,08 \\
Fosfato bicálcico & 1,36 & 1,37 \\
Sal & 0,40 & 0,38 \\
DL-metionina & 0,15 & 0,24 \\
Premix Roche* & 0,30 & 0,30 \\
Semilla de linaza & - & 12,69 \\
& & \\
Análisis calculado & 2930 & 2900 \\
Energía metabolisable (kcal/kg) & 17,00 & 17,00 \\
Proteína bruta & 0,52 & 0,57 \\
Metionina & 0,70 & 0,72 \\
Metionina + Cistina & 4,20 & 4,20 \\
Calcio & 0,56 & 0,59 \\
Fósforo total & 0,35 & 0,34 \\
Fósforo disponible & 2,75 & 3,04 \\
Fibra &
\end{tabular}

(*) Premix vitamínico, mineral y de aminoácidos provee (por kg de dieta): vitamina A, 7950 UI; vitamina D3, 2559 UI; vitamina E, 10,5 mg; vitamina K3, 2,6 mg; riboflavina, $5 \mathrm{mg}$; vitamina B12, $12 \mathrm{mcg}$; ácido nicotínico, $25 \mathrm{mg}$; ácido pantoténico, $8 \mathrm{mg}$; hierro, $50 \mathrm{mg}$; cobre, $10 \mathrm{mg}$; zinc, $50 \mathrm{mg}$; manganeso, $80 \mathrm{mg}$; yodo, $1 \mathrm{mg}$; cobalto, $1 \mathrm{mg}$; selenio, $0,15 \mathrm{mg}$; metionina, $0,9 \mathrm{~g}$; colina, $0,1 \mathrm{~g}$; antioxidante, $30 \mathrm{mg}$.

\section{Experimento}

Fueron utilizadas 192 gallinas ponedoras de linage comercial Babcock de 22 semanas de edad, las cuales fueron divididas en 8 grupos, $(n=24)$. Las aves fueron mantenidas en jaulas individuales y alimentadas $a d$ libitum, durante 30 días.

\section{Muestreo}

El muestreo de los huevos fue realizado durante los 30 días del experimento, dividido en 4 períodos $(0,10,20$ e 30 días). Fueron recogidos 12 huevos por tratamiento, las yemas de huevo fueron liofilizadas y almacenadas en freezer a $-18{ }^{\circ} \mathrm{C}$ hasta la ejecución de los análisis.

Determinación del perfil de ácidos grasos de las yemas liofilizadas

El perfil de ácidos grasos fue determinado por cromatografía gaseosa, utilizándose el método de metilación directa de acuerdo a Wang et al. (2000), siguiendo el siguiente esquema: pesar aproximadamente $50 \mathrm{mg}$ de yema de huevo en tubo de esterificación. Añadir el estándar interno (ácido nonadecanoico C19:0)
2,0 $\mathrm{mg} / \mathrm{mL}$ de hexano. Colocar $1 \mathrm{~mL}$ de metanol y $3 \mathrm{~mL}$ de $\mathrm{HCl}$ metanólico $3 \mathrm{~N}$. Cerrar y ajustar el tubo y llevar a baño maría a $95^{\circ} \mathrm{C}$ por 1 hora. Enfriar a temperatura ambiente y aumentar $8 \mathrm{~mL}$ de una solución de $\mathrm{NaCl}$ 0,88\% y $3 \mathrm{~mL}$ de hexano y mezclar en agitador de tubos. Dejar en reposo hasta separación de las fases. Recoger la fase superior y almacenar en frasco ambar. La muestra está lista para ser inyectada en el cromatógrafo gaseoso, marca Shimadzu, modelo GC17A, con las siguientes condiciones cromatográficas:

Columna: capilar de sílica fundida, Carbowax 20M (30 m x $0,25 \mathrm{~mm} \mathrm{d.i)}$

Detector: ionización de llama

Programación de la columna: temperatura inicial de $70^{\circ} \mathrm{C}$, mantener por $3 \mathrm{~min}$; aumentar hasta $180^{\circ} \mathrm{C}\left(30^{\circ} \mathrm{C} / \mathrm{min}\right)$, mantener por $10 \mathrm{~min}$. Aumentar la temperatura hasta $230{ }^{\circ} \mathrm{C}\left(5^{\circ} \mathrm{C} / \mathrm{min}\right)$, mantener por $15 \mathrm{~min}$.

Gas de arrastre: helio, con flujo de $3 \mathrm{~mL} / \mathrm{min}$.

Temperatura del inyector: $230^{\circ} \mathrm{C}$.

Temperatura del detector: $240{ }^{\circ} \mathrm{C}$.

Los metil ésteres de los ácidos grasos fueron identificados por comparación con los tiempos de retención de 
los estándares. El contenido de los ácidos grasos, expresado en $\mathrm{mg}$ de ácido graso/g de yema, fue calculado de acuerdo a la siguiente fórmula:

$m g / g=\frac{\text { área pico ácido graso } \mathrm{x} \text { concentración estándar interno }(\mathrm{mg} / \mathrm{mL})}{\text { área pico estándar interno } \mathrm{x} \text { peso amostra }(\mathrm{g})}$

Determinación del grado de oxidación de las yemas de huevo

Para evaluar la extensión de la oxidación de los lipídos en las muestras de yemas de huevo, fue realizada la prueba de TBARS (substancias reactivas con el ácido tiobarbitúrico), según Vynckel (1970) y Ramanathan y Das (1992), con algunas adaptaciones de acuerdo al tipo de muestra:

Pesar $5 \mathrm{~g}$ de muestra (yema liofilizada). Disolver con aproximadamente $17 \mathrm{~mL}$ de ácido tricloro acético (TCA) al 7,5\%. Agitar por $10 \mathrm{~min}$, filtrar y llevar el filtrado a un balón volumétrico de $10 \mathrm{~mL}$ para completar el volumen con TCA 7,5\%. Colocar $5 \mathrm{~mL}$ del filtrado en un tubo de ensayo con tapa (por duplicado). Añadir $5 \mathrm{~mL}$ de ácido tiobarbitúrico (TBA) 0,02 M en cada tubo. Agitar y llevar a baño maría hirviente por $40 \mathrm{~min}$. Enfriar los tubos y hacer la lectura a una longitud de onda de $530 \mathrm{~nm}$. Fue realizada una curva estándar con tetraetoxi propano (TEP) a una concentración de $6 \times 10^{-5} \mathrm{M} / \mathrm{mL}$.

\section{Tiempo de almacenamiento de las yemas de huevo}

Las yemas liofilizadas de los huevos correspondientes al tiempo final del experimento (30 días de alimentación de las aves) fueron almacenadas a temperatura ambiente $\left(20-25^{\circ} \mathrm{C}\right)$ por 40 días, divididos en 4 períodos: $0,10,20$ y 40 días, en los cuales fue realizada la determinación de TBARS.

\section{Análisis estadística}

Los resultados de los análisis fueron presentados como media y desvío estándar. Los análisis de varianza (ANOVA) y la comparación de medias a través de las pruebas de Dunnett, fueron realizados utilizando el programa GraphPad Instat, versión 2.01.

\section{RESULTADOS Y DISCUSIÓN}

\section{Perfil de ácidos grasos de la semilla de linaza}

En la Tabla II, están presentados los porcentajes de los diferentes ácidos grasos de la semilla de linaza, pudiéndose constatar la alta cantidad de ácido $\alpha$-linolénico en la semilla oleaginosa.

En la Tabla III se observa el perfil de ácidos grasos en la fracción lipídica de las yemas de huevo de las
TABLA II - Perfil de ácidos grasos de la linaza

\begin{tabular}{ll}
\hline Ácidos grasos & \% área \\
\hline Palmítico $(16: 0)$ & $6,54 \pm 0,01$ \\
Esteárico $(18: 0)$ & $4,95 \pm 0,02$ \\
Oleico $(18: 1 \omega 9)$ & $20,68 \pm 0,03$ \\
Linoleico $(18: 2 \omega 6)$ & $13,78 \pm 0,00$ \\
$\alpha$-Linolénico $(18: 3 \omega 3)$ & $53,18 \pm 0,13$ \\
Eicosanoico $(20: 0)$ & $0,15 \pm 0,00$ \\
\hline
\end{tabular}

gallinas alimentadas com 0 y $12,7 \%$ de semilla de linaza molida durante 30 días, pudiéndose verificar la incorporación significativa de los ácidos grasos poliinsaturados omega 3.

\section{Determinación del grado de oxidación en las yemas de huevo}

Se sabe que los ácidos grasos poliinsaturados, por el hecho de poseer varios dobles enlaces, son susceptibles a oxidación, por lo tanto, las yemas de huevo enriquecidas con los mismos, se vuelven también susceptibles al deterioro lipídico, siendo necesario la protección mediante el uso de antioxidantes. El grado de oxidación de las yemas y la eficacia del uso de estos antioxidantes fueron evaluados a través de la prueba de TBARS, cuyos resultados fueron expresados en mg de malonaldehído, MDA $/ \mathrm{kg}$ de yema liofilizada, siendo esta determinación la más usada en trabajos científicos en los cuales se evalúa el grado de peroxidación de la fracción lipídica, tanto de alimentos como de tejidos animales (Nam, Ahn, 2003; Lee, Dabrowski, 2003; Lee et al., 2003; Franchini et al., 2002; Skrivanova et al., 2001; Yang, Chen, 2001).

Los resultados de esta prueba (Tabla IV), para el tratamiento con $0 \%$ de aceite de linaza, revelaron disminución de los valores de MDA tanto en los antioxidantes sintéticos como en los naturales, en los tiempos 10, 20 y 30 días con relación a su respectivo control, siendo esta diferencia significativa. Para el tratamiento con $5 \%$ de aceite de linaza, se verificó diferencia significativa $(\mathrm{p}<0,001)$ en la reducción de los valores de MDA en los tiempos 20 y 30 días, para los 3 antioxidantes, como también para el BHA+BHT en el tiempo 10 días. Es necesario destacar que en los grupos control huvo aumento en el valor de la absorbancia en el transcurso del tiempo, sin embargo, en los tratamientos con antioxidantes esta característica fue inversa. Se observó también que en el tiempo 0 (cero) en las 2 dietas (0 y $12,7 \%$ de semilla de linaza) para todos los antioxidantes, los valores de MDA fueron más altos que el control. 
TABLA III - Perfil de ácidos grasos en yemas liofilizadas de huevos enriquecidos con ácidos grasos omega 3

\begin{tabular}{lcc}
\hline ÁCIDO GRASO & $\begin{array}{c}\text { CONTROL } \\
\text { (0\% semilla de linaza) }\end{array}$ & $\begin{array}{c}\text { MUESTRA } \\
\mathbf{( 1 2 , 7 \%} \text { semilla de linaza) }\end{array}$ \\
\hline Mirístico: $(14: 0)$ & $0,18 \pm 0,02$ & $0,16 \pm 0,01$ \\
Palmítico: $(16: 0)$ & $28,41 \pm 0,62$ & $26,46 \pm 0,83$ \\
Palmitoleico: $(16: 1)$ & $1,03 \pm 0,06$ & $1,39 \pm 0,08$ \\
Heptadecanoico: $(17: 0)$ & $0,12 \pm 0,02$ & $0,14 \pm 0,03$ \\
Esteárico: $(18: 0)$ & $12,75 \pm 0,33$ & $13,96 \pm 0,58$ \\
Oleico: $(18: 1 \omega 9)$ & $30,86 \pm 0,38$ & $31,79 \pm 0,61$ \\
Linoleico: $(18: 2 \omega 6)$ & $18,30 \pm 0,62$ & $14,75 \pm 0,49$ \\
$\alpha$-Linolénico: $(18: 3 \omega 3)$ & $0,23 \pm 0,03$ & $3,75 * * * \pm 0,24$ \\
Eicosenoico: $(20: 1)$ & $0,14 \pm 0,01$ & $0,10 \pm 0,01$ \\
Araquidónico: $(20: 4 \omega 6)$ & $4,92 \pm 0,09$ & $2,27 \pm 0,11$ \\
Behénico: $(22: 0)$ & $0,12 \pm 0,01$ & $0,10 \pm 0,01$ \\
Docosadienoico: $(22: 2)$ & $0,46 \pm 0,02$ & $0,40 \pm 0,03$ \\
Docosahexaenoico: $(22: 6 \omega 3)$ & $1,77 \pm 0,11$ & $4,35 * * * 0,24$ \\
\hline$\sum$ AG Sat. & 41,58 & 40,82 \\
$\sum$ AG Monoinsat. & 32,09 & 33,28 \\
$\sum$ AG Poliinsat. & 25,66 & 25,52 \\
$\sum$ AG $\omega-6$ & 23,22 & 17,02 \\
$\sum$ AG $\omega-3$ & 2,00 & 8,10 \\
$\omega-6$ / $\omega-3$ & 11,61 & 2,10 \\
\hline
\end{tabular}

*** Media difiere estadísticamente del control a nivel de error de $0,1 \%(\mathrm{p}<0,001)$.

Es importante destacar que los valores de MDA de todos los tratamientos de la dieta con $12,7 \%$ de semilla de linaza en los diferentes tiempos, fueron mayores cuando comparados con los tratamientos con $0 \%$ de linaza. Estos resultados sugieren que el mayor número de insaturación del ácido $\alpha$-linolénico (LNA, $\omega$-3) presente en la linaza, provocó un mayor grado de oxidación.

En general, en las 2 dietas ( 0 y $12,7 \%$ de semilla de linaza), en los 3 períodos de tiempo la presencia de los antioxidantes naturales y sintéticos mostró protección

TABLA IV - Prueba de TBARS en las yemas liofilizadas de huevo de los tratamientos con 0 y $12,7 \%$ de semilla de linaza con su respectivo control, sin antioxidante, en los 4 períodos de tiempo, expresados en $\mathrm{mg}$ de MDA/kg yema liofilizada

\begin{tabular}{lcccc}
\hline $\mathbf{0 \%}$ LINAZA & TIEMPO 0 & TIEMPO 10 & TIEMPO 20 & TIEMPO 30 \\
\hline CONTROL & $0,86 \pm 0,00$ & $0,97 \pm 0,01$ & $1,03 \pm 0,01$ & $0,97 \pm 0,01$ \\
BHA + BHT & $0,97 * * * \pm 0,00$ & $0,80^{* * *} \pm 0,00$ & $0,86^{*} \pm 0,02$ & $0,75^{* * *} \pm 0,01$ \\
ORÉGANO & $0,97 * * * \pm 0,00$ & $0,86^{* * *} \pm 0,01$ & $0,86^{*} \pm 0,01$ & $0,80^{* * *} \pm 0,01$ \\
ROMERO & $1,03^{* * *} \pm 0,01$ & $0,92^{* *} \pm 0,01$ & $0,92^{\text {ns }} \pm 0,01$ & $0,80^{* * *} \pm 0,02$ \\
\hline $\mathbf{1 2 , 7 \%}$ LINAZA & TIEMPO 0 & TIEMPO 10 & TIEMPO 20 & TIEMPO 30 \\
\hline CONTROL & $0,92 \pm 0,00$ & $1,03 \pm 0,00$ & $1,09 \pm 0,01$ & $1,15 \pm 0,01$ \\
BHA + BHT & $1,03^{* *} \pm 0,01$ & $0,97^{* * *} \pm 0,00$ & $0,97 * * \pm 0,01$ & $0,97^{* * *} \pm 0,00$ \\
ORÉGANO & $1,03^{* * *} \pm 0,00$ & $1,03^{\text {ns }} \pm 0,00$ & $0,92^{* * *} \pm 0,01$ & $1,03^{* *} \pm 0,01$ \\
ROMERO & $0,97^{\text {ns }} \pm 0,01$ & $1,03^{\text {ns }} \pm 0,00$ & $0,92^{* * *} \pm 0,01$ & $0,97^{* * *} \pm 0,01$ \\
\hline
\end{tabular}

Tiempo expreso en días; ns media no difiere estadísticamente del control 0 (cero) día ( $>0,05) ; *$ media difiere estadísticamente del control 0 día a un nivel de error de $5 \%(\mathrm{p}<0,05) ; * *$ media difiere estadísticamente del control 0 día a un nivel de error de $1 \%(\mathrm{p}<0,01)$; ${ }^{* * *}$ media difiere estadísticamente del control 0 día a un nivel de error de $0,1 \%$ $(\mathrm{p}<0,001)$ 
considerable contra la oxidación lipídica, comprobándose así, su efecto antioxidante en el modelo experimental utilizado en este trabajo.

En el estudio realizado por Aymond y Van Elswyk (1995), fueron determinados los valores de las substancias reactivas al ácido tiobarbitúrico (TBARS) expresados em $\mathrm{mg}$ de TBARS/kg de yema. Fueron probados huevos enriquecidos con PUFA $\omega-3$ a través de dietas suplementadas con 5 y $15 \%$ de semilla de linaza entera e molida durante 5 semanas. Em este ensayo no fue encontrada diferencia significativa entre los valores de TBARS de los tratamientos $(0,20-0,23 \mathrm{mg} / \mathrm{kg}$ de yema $)$ en relación al control $(0,18 \mathrm{mg} / \mathrm{kg}$ de yema). Con todo, en nuestro trabajo encontramos diferencias estadísticamente significativas entre los tratamientos con 0 y $5 \%$ de aceite de linaza, revelando por lo tanto, que la peroxidación lipídica ocurrió por la presencia de la mayor cantidad de ácidos grasos poliinsaturados.

Para Qi y Sim (1998) el uso de tocoferol natural (0, 200,400 y $800 \mathrm{mg} / \mathrm{kg}$ de dieta) en dietas suplementadas con $15 \%$ de linaza y $0,5 \%$ de aceite de pescado, llevó a la reducción significativa de los valores de TBARS de 41,32 a 18,57 nmol de malonaldehído/g de yema. Esta protección contra la oxidación, por parte del antioxidante, observada por los mencionados autores, apoyan nuestros resultados, los cuales sugieren que la estabilidad lipídica de los huevos enriquecidos con PUFA $\omega-3$ puede ser mejorada por la incorporación de antioxidantes, tanto sintéticos como naturales.

A través de los valores de MDA presentados en la Tabla V, puede ser verificado una vez más la actuación de los antioxidantes naturales en la protección contra la oxidación lipídica en las yemas de huevo sometidas a almacenamiento por un período de 40 días. Según estos valores se puede deducir que el grado de oxidación lipídica está en función del tiempo, así, de forma general, huvo aumento de los valores de MDA en el transcurso del tiempo, cuando comparados con el tiempo 0 (cero) día, ocurriendo mayor oxidación en el tiempo final (40 días). Podemos observar también la actuación de los antioxidantes tanto sintéticos como naturales en la protección contra la oxidación lipídica, presentando diferencias significativas $(p<0,01)$ cuando relacionados con su respectivo control. Esta respuesta se constata a partir del tiempo 10 días de alimentación de las aves. Es importante destacar los valores más altos de MDA para los tratamientos con $5 \%$ de aceite de linaza cuando comparados con $0 \%$ de aceite, a partir del tiempo 10 días de alimentación, verificándose así la mayor susceptibilidad de los ácidos grasos poliinsaturados al deterioro lipídico.

Los efectos benéficos para la salud, ofrecidos por los PUFA $\omega-3$ incorporados en las yemas de huevo, pueden estar limitados por la calidad oxidativa. Marshall et al. (1994) determinaron la estabilidad de huevos con cáscara, de gallinas alimentadas con $1,5 \%$ de aceite de menhaden, almacenados a temperatura de refrigeración durante 4 semanas. Los autores indicaron que, aunque los valores de TBARS en las yemas no hubiesen aumentado durante el almacenamiento de los huevos con cáscara, los TBARS fueron mayores en la yema de los huevos con aceite de menhaden quando comparados con el control. Estos resultados no están en conformidad con os encontrados en nuestro trabajo, en el cual se verificó aumento de la oxidación en el transcurso del tiempo de almacenamiento de las yemas de huevo a temperatura ambiente $\left(20-25^{\circ} \mathrm{C}\right)$. Sin embargo, fue observada diferencia significativa en el efecto de la dieta, como fue encontrado por Marshall et al. (1994), que sugieren que la presencia de TBARS puede ser

TABLA V - Prueba de TBARS en las yemas liofilizadas almacenadas durante 40 días a $20-25^{\circ} \mathrm{C}$, correspondientes al tiempo final del experimento, expresados en $\mathrm{mg}$ de $\mathrm{MDA} / \mathrm{kg}$ de yema liofilizada

\section{TIEMPO DE ESTABILIDAD (días)}

\begin{tabular}{lcccc} 
TRATAMIENTO & $\mathbf{0}$ & $\mathbf{1 0}$ & $\mathbf{2 0}$ & $\mathbf{4 0}$ \\
\hline Control 0\% linaza & $1,09 \pm 0,00$ & $1,09 \pm 0,00$ & $1,15 \pm 0,01$ & $1,72 \pm 0,01$ \\
BHA+BHT & $0,75^{* *} \pm 0,00$ & $0,80 * * \pm 0,01$ & $0,80 * * \pm 0,01$ & $1,09 * * \pm 0,00$ \\
Orégano & $0,80 * * \pm 0,01$ & $0,86^{* *} \pm 0,01$ & $0,86^{* *} \pm 0,01$ & $0,97 * * \pm 0,01$ \\
Romero & $1,03^{\mathrm{ns}} \pm 0,00$ & $1,03 * * \pm 0,01$ & $0,97 * * \pm 0,01$ & $1,43 * * \pm 0,01$ \\
Control 12,7\% linaza & $1,20 \pm 0,01$ & $1,20 \pm 0,01$ & $1,32 \pm 0,01$ & $1,83 \pm 0,01$ \\
BHA+BHT & $0,97^{* *} \pm 0,00$ & $0,92^{* *} \pm 0,00$ & $1,09 * * \pm 0,01$ & $1,43 * * \pm 0,01$ \\
Orégano & $1,38^{* *} \pm 0,01$ & $1,15^{\mathrm{ns}} \pm 0,01$ & $1,15^{* *} \pm 0,01$ & $1,49 * * \pm 0,01$ \\
Romero & $1,20^{\mathrm{ns}} \pm 0,01$ & $1,15^{\mathrm{ns}} \pm 0,01$ & $1,15^{* *} \pm 0,01$ & $1,66 * * \pm 0,01$ \\
\hline
\end{tabular}

${ }^{n s}$ Media no difiere estadísticamente del control $(\mathrm{p}>0,05) ; * *$ Media difiere estadísticamente del control a nivel de error de $1 \%(\mathrm{p}<0,01)$ 
debido al consumo y consiguiente incorporación de productos de la oxidación lipídica por parte de las aves o la producción in vivo de TBARS en los hígados de las aves alimentadas con dietas altamente insaturadas, para después ser transportados y depositados en las yemas de los huevos.

Aymond y Van Elswyk (1995) mencionan que la protección lipídica parcial dada por la cubierta de la semilla de linaza intacta podría reducir el potencial para la producción de huevos conteniendo lípidos oxidados, por lo tanto, el uso de la semilla entera en las dietas de las aves resultaria en huevos enriquecidos con PUFA $\omega-3$ con valores de TBARS reducidos cuando son comparados con la semilla molida y/o aceite de pescado. Sin embargo, la utilización de linaza molida en las dietas de las aves, favorece en la digestión y absorción del aceite.

Se sabe que los productos de la oxidación lipídica, como aldehídos, cetonas, hidroxiácidos, entre otros, pueden ocasionar daño de tejidos biológicos, por lo tanto, cabe indicar que es necesario considerar la estabilidad oxidativa de los productos enriquecidos con PUFA, entre ellos, los huevos $\omega$-3, estabilidad que puede ser alcanzada a través de la utilización de antioxidantes tanto sintéticos como naturales.

\section{CONCLUSIÓN}

Mediante el uso de la semilla de linaza en la ración de gallinas ponedoras se obtuvieron huevos con alta concentración de ácidos grasos poliinsaturados y por lo tanto susceptibles de oxidación. La actividad antioxidante de los extractos de los condimentos romero y orégano aumentó la estabilidad de los huevos enriquecidos con PUFA $\omega-3$.

\section{RESUMO}

\section{Estabilidade oxidativa de ovos enriquecidos com ácidos graxos poliinsaturados ômega 3 , frente a antioxidantes naturais}

Foram alimentadas 192 galinhas poedeiras de 22 semanas de idade da linhagem comercial Babcock, durante 30 dias com dietas constituidas de 0 (zero) e 12,7\% de semente de linhaça moída correspondente a 0 e $5 \%$ de óleo de linhaça, respectivamente. Foram definidos 8 tratamentos: 4 grupos com 5\% de óleo de linhaça (controle/sem antioxidante; $B H A+B H T, 100+100$ ppm; orégano, 200 ppm; alecrim, 200 ppm) e 4 grupos sem óleo de linhaça, mas utilizando os mesmos antioxidantes. Este estudo foi realizado com o objetivo de verificar a eficácia do uso de antioxidantes naturais provenientes do orégano e do ale- crim, na proteção contra a deterioração oxidativa da fração lipídica das gemas dos ovos enriquecidos com ácidos graxos poliinsaturados ômega 3 (PUFA $\omega$-3). O grau de oxidação lipídica foi determinado através do teste TBARS (substâncias reativas com ácido tiobarbitúrico). De acordo aos resultados obtidos, verificou-se diferença significativa na redução do grau de oxidação lipídica das gemas de ovo em todos os tratamentos com antioxidantes, quando relacionados ao seu respectivo controle. Portanto, os extratos das especiarias, alecrim e orégano, podem ser utilizados satisfatoriamente para se obter ovos enriquecidos com PUFA $\omega-3$, melhorando a estabilidade lipídica.

UNITERMOS: Peroxidação lipídica. Ácidos graxos poliinsaturados ômega 3. Antioxidantes naturais.

\section{AGRADECIMIENTOS}

Agradecemos a FAPESP y al CNPq por el apoyo financiero y a CAPES por la beca de investigación en el programa PEC-PG.

\section{REFERENCIAS BIBLIOGRÁFICAS}

AYMOND, W.M.; VAN ELSWYK, M.E. Yolk thiobarbituric acid reactive substances and n-3 fatty acids in response to whole and ground flaxseed. Poult. Sci., Savoy, v. 74, p. 1388-1394, 1995.

BERNAL-GÓMEZ. M.E.; DELLA TORRE, J.; RODAS, M.A.; MENDONÇA-JÚNIOR, C.X.; MANCINIFILHO, J. Avaliação sensorial e instrumental de ovos de galinhas alimentadas com rações suplementadas com óleo de linhaça e antioxidantes. Nutrire: Soc. Bras. Alim. Nutr., São Paulo, v. 23, p. 55-66, 2002.

CINTRA, R.M.G.; MANCINI-FILHO, J. Efeito antioxidante de especiarias: avaliação e comparação de métodos in vitro e in vivo. Nutrire: Soc. Bras. Alim. Nutr., São Paulo, v. 22, p. 49-62, 2001.

FRANCHINI, A.; SIRRI, F.; TALLARICO, N.; MINELLI, G.; IAFFALDANO, N.; MELUZZI, A. Oxidative stability and sensory and functional properties of eggs from laying hens fed supranutritional doses of vitamins E and C. Poult. Sci., Savoy, v. 81, n. 11, p. 1744-1750, 2002.

HARTMAN, L.; LAGO, B.C.A. Rapid preparation of fatty acid methyl esters from lipids. Lab. Pract., London, v. 22, p. $475-477,1973$. 
LEE, K.J.; DABROWSKI, K. Interaction between vitamins $\mathrm{C}$ and $\mathrm{E}$ affects their tissue concentrations, growth, lipid oxidation, and deficiency symptoms in yellow perch (Perca flavescens). Br. J. Nutr., Wallingford, v. 89, n. 5, p. 589-596, 2003.

LEE, S.R.; IM, K.J.; SUH, S.I.; JUNG, J.G. Protective effect of green tea polyphenol (-)-epigallocatechin gallate and other antioxidants on lipid peroxidation in gerbil brain homogenates. Phytother. Res., London, v. 17, n. 3, p. 206-209, 2003.

LOPEZ BOTE, C.J.; SANZ ARIAS, R.; REY, A.I.; CASTAÑO, A.; ISABEL, B.; THOS, J. Effect of freerange feeding on $\mathrm{n}-3$ fatty acid and a-tocopherol content and oxidative stability of eggs. Anim. Feed Sci. Technol., Amsterdam, v. 72, p. 33-40, 1998.

MADSEN, H.L.; BERTELSEN, G. Spices as antioxidants. Trends Food Sci. Technol., Oxford, v. 6, p. 271-277, 1995.

MARSHALL, A.C.; SAMS, A.R.; VAN ELSWYK, M.E. Oxidative stability and sensory quality of stored eggs from hens fed 1,5\% menhaden oil. J. Food Sci., Chicago, v. 59, n. 3, p. 561-563, 1994.

MEYDANI, S.N. Effect of (n-3) polyunsaturated fatty acids on cytokine production and their biologic function. Nutrition, New York, v. 12, p. S8-S14, 1996.

NAM, K.C.; AHN, D.U. Use of double packaging and antioxidant combinations to improve color, lipid oxidation, and volatiles of irradiated raw and cooked turkey breast patties. Poult. Sci., Savoy, v. 82, n. 5, p. 850857, 2003.

NAMIKI, M. Antioxidants/antimutagens in food. Crit. Rev. Food Sci. Nutr., Lauderdale, v. 29, n. 4, p. 273-300, 1990.

NATIONAL RESEARCH COUNCIL. Subcommittee on Poultry Nutrition. Committee on Animal Nutrition. Nutrient requirements of poultry. 9.ed. Washington: National Academy Press, 1994. 155p.

NAWAR, W.W. Lipids. In: FENNEMA, O.R. Food chemistry. 3.ed. New York: Marcel Dekker, 1996. p. 225319. (Food science and technology).
QI, G.H.; SIM, J.S. Natural tocopherol enrichment and its effect in n-3 fatty acid modified chicken eggs. J. Agric. Food Chem., Columbus, v. 46, p. 1920-1926, 1998.

RAMANATHAN, L.; DAS, N.P. Studies on the control of lipid oxidation in ground fish by some polyphenolic natural products. J. Agric. Food Chem., Columbus, v. 40, p. 17-21, 1992.

SHAHIDI, F.; WANASUNDARA, P.K.J.P.D. Phenolic antioxidants. Crit. Rev. Food Sci. Nutr., Lauderdale, v. 32, n. 1, p. 67-103, 1992.

SILVA, F.A.M.; BORGES, M.F.M.; FERREIRA, M.A. Métodos para avaliação do grau de oxidação lipídica e da capacidade antioxidante. Quim. Nova, São Paulo, v. 22, n. 1, p. $94-103,1999$.

SKRIVANOVA, V.; SKRIVAN, M.; MAROUNEK, M.; BARAN, M. Effect of feeding supplemental copper on performance, fatty acid profile and on cholesterol contents and oxidative stability of meat of rabbits. Arch. Tierernaehr., Berlin, v. 54, n. 4, p. 329-339, 2001.

VYNCKE, W. Direct determination of the thiobarbituric acid value in trichloracetic acid extracts of fish as a measure of oxidative rancidity. Fette-Seifen Anstrichmittel, Hamburgo, v. 72, n. 12, p. 1084-1087, 1970.

WANASUNDARA, U.N.; SHAHIDI, F. Antioxidant and pro-oxidant activity of green tea extracts in marine oils. Food Chem., Kidlington, v. 63, n. 3, p. 335-342, 1998.

WISEMAN, H. Dietary influences on membrane function: importance in protection against oxidative damage and disease. J. Nutr. Biochem., Stoneham, v. 7, p. 2-15, 1996.

YANG, S.C.; CHEN, K.H. The oxidation of cholesterol in the yolk of selective traditional Chinese egg products. Poult. Sci., Savoy, v. 80, n. 3, p. 370-375, 2001.

Recebido para publicação em 07 de abril de 2003. 